\title{
Spin down of an Accreting Millisecond Pulsar, the case of XTE J1814-338
}

\author{
A.Papitto*, T.Di Salvo ${ }^{\dagger}$, L.Burderi $^{* *}$, M.T.Menna ${ }^{\ddagger}$, G.Lavagetto $^{\dagger}$ and \\ A.Riggio** \\ *Dipartimento di Fisica, Universitá degli Studi di Roma "Tor Vergata”, via della Ricerca \\ Scientifica 1,00133, Roma, Italy, email: papitto@oa-roma.inaf.it \\ †ipartimento di Scienze Fisiche ed Astronomiche, Università di Palermo, via Archirafi 36. \\ Palermo, 90123, Italy \\ ${ }^{* *}$ Dipertimento di Fisica, Universitá degli Studi di Cagliari, SP Monserrato-Sestu, KM 0.7, \\ Monserrato, 09042 Italy \\ ¥Osservatorio Astronomico di Roma, via Frascati 33, Monteporzio Catone, 00040, Italy
}

\begin{abstract}
.
We report about a timing analysis performed on the data gathered by RXTE of the accreting millisecond pulsar XTE J1814-338 during its 2003 outburst. The first full orbital solution of this binary system is given. Moreover the evolution of the phase of the pulsed emission reveals that the rotating compact object is spinning down at a rate $\dot{v}=(-6.7 \pm 0.7) \times 10^{-14} \mathrm{~Hz} / \mathrm{s}$, while accreting. This behavior is considered as a result of the braking effect due to the interaction between the magnetosphere and the inner parts of the accretion disc, in the case of an accretion rate low enough to allow the expansion of the magnetospheric radius to the corotation limit. In this context we derive an estimate of the superficial magnetic field, $B_{S} \simeq 8 \times 10^{8} \mathrm{G}$, which lies in the plausible range for the accreting millisecond pulsars to be the progenitors of the radio millisecond pulsar. We also report about a modulation of the phase delays around the mean timing solution, which we interpret as a signature of movements of the accreting hotspot, resulting from a variable accretion rate.
\end{abstract}

Keywords: stars: neutron - stars: magnetic fields - pulsars: general - pulsars: individual: XTE J1814-338 - X-ray: binaries

PACS: 97.10.Gz, 97.60.Gb, 97.60.Jd, 97.80.Jp

\section{INTRODUCTION}

Accreting Millisecond Pulsars (hereafter AMSP) are neutron stars harbored in a Low Mass X-ray Binary (hereafter LMXB), which, during accretion of matter, show coherent modulation of their X-ray emission with a periodicity of the order of few ms. Objects of this kind were long sought as plausible progenitors of the millisecond radio pulsars, according to the recycling scenario (see e.g. [1]). The basic idea is that an old, weakly magnetized and presumably slowly spinning neutron star, harbored in a binary system, can be spun up by accretion of matter and angular momentum from a (Keplerian) accretion disc, down to spin periods of the order of few milliseconds. At the end of this long accretion phase the neutron star is so rapidly spinning that it may become able to switch on again the mechanism that drives the radio pulsar phenomenon, therefore appearing as a millisecond radio pulsar.

Only recently the large collecting capabilities and the unprecedented temporal resolution of Rossi X-ray Timing Explorer $(R X T E)$ allowed the discovery of an X-ray tran-

CP924, The Multicolored Landscape of Compact Objects and their Explosive Origins - Cefalù 2006, edited by T. Di Salvo, L. A. Antonelli, G. L. Israel, L. Piersanti, A. Tornambè, L. Burderi, F. Fiore, G. Matt, and M. T. Menna (C) 2007 American Institute of Physics 978-0-7354-0434-2/07/\$23.00 
sient, whose outburst light curve was coherently modulated with $P_{\text {spin }}=2.5 \mathrm{~ms}$, SAX J1808.4-3658 [2]. Seven accreting millisecond pulsar have been discovered so far (see [3] for a review), they have spin periods ranging from $\sim 1.5 \mathrm{~ms}$ to $\sim 5.5 \mathrm{~ms}$, and they are all harbored in very compact binaries $\left(P_{\text {orb }}=0.7-4.3 \mathrm{hr}\right)$, with very low mass companions $\left(\lesssim 0.15 M_{\odot}\right)$.

In this paper we report about the first precision timing analysis of XTE J1814-338, performed to evaluate the balance of the positive torque due to the accretion of matter and the negative torque due to the interaction between the NS magnetic field and the incoming matter in the disc.

\section{OBSERVATIONS AND DATA ANALYSIS}

XTE J1814-338 was detected in outburst on 2003 June 5 [4] and was extensively monitored by RXTE until it switched back to quiescence, 53 days later. The RXTE/PCA $2.5-25 \mathrm{keV}$ light curve is plotted in the upper panel of Fig.1; the observed peak $2.5-25 \mathrm{keV}$ flux is $5 \times 10^{10} \mathrm{erg} / \mathrm{cm}^{2} / \mathrm{s}$, while the light curve shape consists of a $\sim 30$ d plateau, during which three bumps appear with variations of $\sim 20 \%$ of the observed flux, followed by an abrupt cut off to one forth of the previous average luminosity, after which the source entered in quiescence.

We considered all the publicly available observation of XTE J1814-338, performed by the RXTE Proportional Counter Array (PCA, [5]) in the period 2006 June 7 to July 27. We considered data taken in Event Mode with 64 energy channels and a $125 \mu$ s temporal resolution. We first corrected the photon arrival times for the motion of the spacecraft in the Solar System, by using JPL DE-405 ephemerides and the optical counterpart position, that has a $90 \%$ confidence radius of $0^{\prime \prime} .2[6]$.

Coherent pulsations were observed for the whole course of the outburst, by folding the light curve around the spin period $3.18110566967 \mathrm{~ms}$. The resulting pulse profiles were efficiently modeled with two sinusoidal components. The significance of each detection was estimated by considering the ratio between the fractional amplitude of the considered harmonic component and the respective uncertainty; while the fundamental frequency was detected at a high degree of significance in nearly all the observations, the second harmonic significance fell below the $2 \sigma$ threshold $35 \mathrm{~d}$ since the first observation considered, thus limiting the temporal base line on which perform the timing analysis.

The potentiality of the timing technique in reproducing an accurate picture of the spin behavior of a neutron star, resides in the estimation of the time of arrival of a given pulse and in its comparison with the one predicted by using a certain guess on the rotational and orbital behavior of the source, namely the residual, $R_{\phi}(t)=\phi_{\text {pred }}(t)-\phi_{\text {obs }}(t)$. The timing technique we applied in the case of XTE J1814-338 is extensively described in [7].

Before all we considered the effects of the orbital motion on the arrival times. The orbital solution we obtain can be summarized by four parameters, namely, the orbital period, $P_{O R B}=15388.7229(2) \mathrm{s}$, the semimajor axis, $a \operatorname{sini} / c=390.633(9)$ lt-ms, the epoch of passage of the NS at the ascending node, $T^{*}=52797.8101689(9)$ MJD and the eccentricity, $e<2.4 \times 10^{-5}$ ( $3 \sigma$ upper limit). The uncertainties, given above in parentheses, are quoted at the 90 per cent confidence level, as in the rest of the paper. 


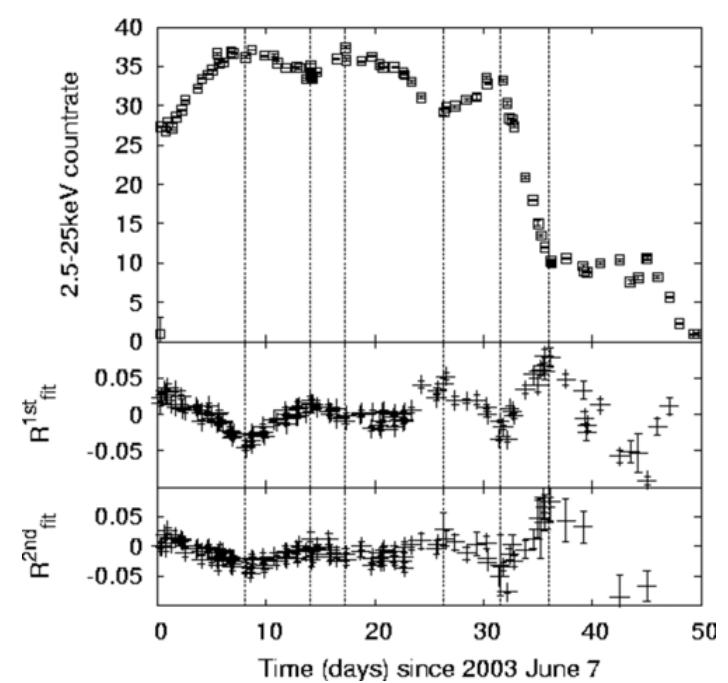

FIGURE 1. (2.5-25keV) light curve of the 2003 outburst of J1814, as taken by the PCA's Unit 2 aboard RXTE. Each point represents the average count rate in every available observation, which were preliminarily background subtracted according to the faint source model (top panel). In the lower panels we plot residuals in their natural units from the best-fitting constant spin down model of the fundamental $\left(R_{f i t}^{1 s t}\right.$, middle panel) and of the second harmonic $\left(R_{f i t}^{2 n d}\right.$, bottom panel) phase delays. These plots are the same as lower panels of Fig.2, and are plotted here to highlight the correlation between the shape of the light curve and the modulation that affects phase residuals.

After having corrected all the photon arrival times for the orbital effects, we could focus on the phase delays as affected only by the torques acting on the NS. The residuals are then expected to follow the relation, $R_{\phi}(t)=-\phi_{0}-\delta v t-\delta \phi_{\dot{v}}(t)$, where $\phi_{0}$ is a constant, $\delta v$ is the correction to the frequency at the beginning of observations and $\delta \phi_{\dot{v}}(t)$ is the phase variation induced by a spin frequency derivative, which in the case of a constant negative $\dot{v}$ reduces to a quadratically increasing term.

This simplified timing procedure is possible because, in the case of AMSP, the different time scales over which the orbital motion (few hours) and the genuine spin evolution (few weeks) have their effect on residuals allow to decouple these two effects. Once the times of arrival of X-ray photons are corrected with our best orbital solution, the remaining uncertainties owing to the orbital motion are normally distributed around the average timing solution, and together with the statistical uncertainties on phase determination, concurs to build the overall uncertainties on phases.

On the other hand the uncertainty on the source position makes the residuals oscillate around the average solution with a periodicity $P_{\oplus}$, a time scale far longer than the typical outburst duration, so that corrections on the position cannot be determined with such a short baseline. Nevertheless [7] also showed how the expression governing the delays induced by a slightly wrong position can be used to derive an upper limit on the effects of uncertainties in source coordinates over the spin parameters obtained with a timing analysis. The procedure there outlined allows to derive the systematic errors affecting 

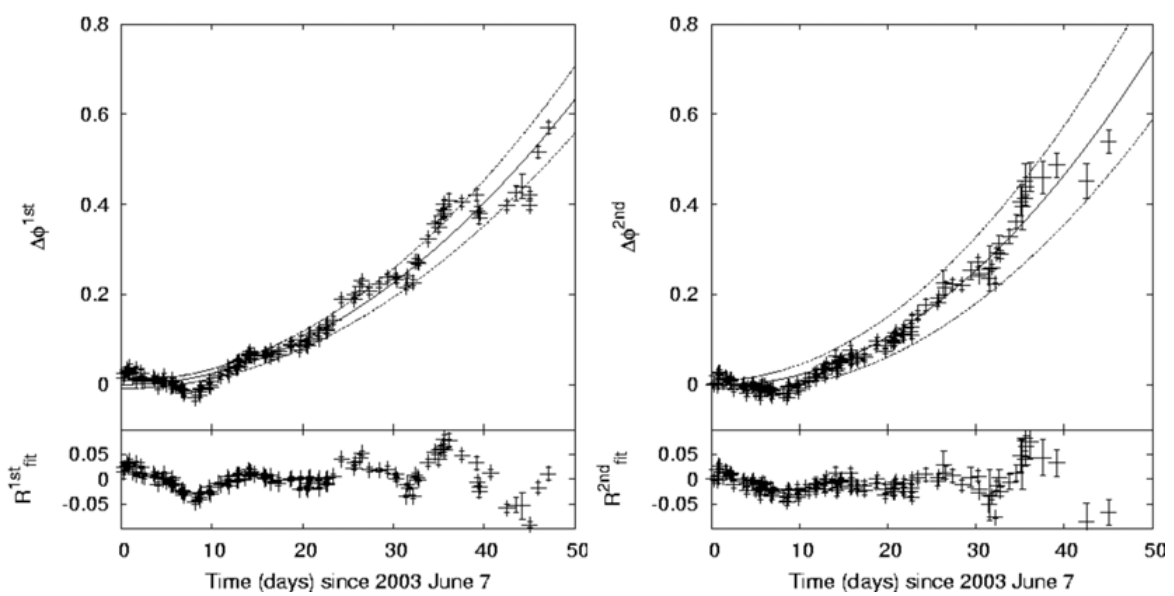

FIGURE 2. In the left panel we plot the evolution of the pulse phase delays, measured in their natural units, computed upon the fundamental frequency component, folding every available observation around $P=3.18110566967 \mathrm{~ms}$. The solid line is the best fit constant $\dot{v}$ model, while the dashed lines represent the contours of the $90 \%$ confidence region (upper panel). Residuals in the same units with respect to this model (lower panel). In the right panel we plot the same quantities, this time referred to the timing of the second harmonic component

the spin frequency correction and the spin frequency derivative. In the case of XTE J1814-338 we have $\sigma_{\text {syst } \Delta v} \leq 3 \times 10^{-8} \mathrm{~Hz}$ and $\sigma_{\text {syst } \dot{v}} \leq 6 \times 10^{-15} \mathrm{~Hz} / \mathrm{s}$.

The considered observations were then folded around our best estimate of the spin period; the temporal evolution of the phase delays computed upon the fundamental frequency and the second harmonic are showed in left and right panel of Fig.2 respectively. The best estimate of the initial spin frequency is $v_{0}=314.35610879(1) \mathrm{Hz}$. The general trend followed by the phase delays in both cases is a clear spin down, superimposed on which $\mathrm{a} \sim 12$ days modulation appears. Fitting the phase delays with a constant $\dot{v}$ model we estimate a spin frequency derivative, $\dot{v}^{1 s t}=(-6.7 \pm 0.7) \times 10^{-14} \mathrm{~Hz} / \mathrm{s}$ having considered the fundamental frequency, and $\dot{v}^{2 n d}=(-8.5 \pm 0.9) \times 10^{-14} \mathrm{~Hz} / \mathrm{s}$ for the second harmonic. The discrepancy between these values of $\dot{v}$ in the two cases can be fully explained by the difference of the number of available points. As a matter of fact considering an interval in which the two data sets exactly overlap we obtain values that are fully compatible among the uncertainties.

As the modulation around the mean solution determines poor quality fit, we computed the uncertainties by a least square fit of the phase delays evolution plots, where the single errors affecting each measured phase were amplified by the factor needed to yield a reduced chi squared equal to unity. We believe that this procedure is appropriate to give a reasonable estimates of the uncertainties affecting the measured spin parameters, $v_{0}$ and $\dot{v}$, in presence of residuals that are not normally distributed around the mean solution, nor can be explained in terms of a bad position or orbital solution (see below). 


\section{DISCUSSION AND CONCLUSIONS}

The phase jiggle that crucially affects the quality of the fit performed on the phase delays appears as $\mathrm{a} \sim 12$ days modulation around the average timing solution, (see bottom panels of Fig. 2 for the residuals from the best-fitting constant $\dot{v}$ model). This time-scale rules out the possibility that this modulation could be due to a bad position or a wrong orbital solution, as these would make the phase delays oscillate on very different timescales ( $P_{\oplus}$ and $P_{O R B}$ respectively).

A striking anti-correlation between the shape of the residuals from the best-fitting constant $\dot{v}$ model of the phase delays and the shape of the X-ray flux can be found instead, as it can be seen by following vertical dashed lines in Fig.1. When the flux increases the phases decrease as the source would be spinning up, and the opposite happens when the flux decreases. A linear correlation test yields a Spearman coefficient $R=0.80$, which implies for $N=113$ points a probability of less than 0.01 per cent for the points to be uncorrelated.

This anti-correlation cannot be understood in terms of a variable torque acting on the $\mathrm{NS}$, as a consequence of variations of the instantaneous accretion rate. This is because every vertex in the phase delays evolution (see for example the behavior around day 8 in bottom panel of Fig.1) would imply changes of the spin frequency taking place on time-scales shorter than the sampling one. We argue instead that this modulation reflects movements of the accreting hotspot and the accretion column, being the geometry of the latter controlled by the matter density near the inner edge of the disc. As a matter of fact the radius where the disc is truncated, the magnetospheric radius, is defined by the balance of the ram pressure of the infalling matter and the magnetic pressure of the magnetosphere (see e.g. [8]; [9]). We can then expect that if the magnetospheric radius is reasonably close to the NS surface, as in AMSPs, variations in the density profile near the disc inner edge may become able to bend at some level the magnetic field lines that lead towards the accretion spots. This could be particularly the case for a pulsar that spins down while accreting, as in this case the density profile near the inner edge of the disc is steepened by the request of the overall angular momentum to be transported outward [10]. Moreover if the spin down is caused by the threading of the disc by the magnetic field, this would ensure a close link between matter orbiting in the disc and the lines that lead falling particles to the hotspot. We note that a complex behavior of the phase delays is also showed by at least another AMSP, SAX J1808.4-3658, during its 2002 outburst [11].

Apart from this modulation, the general trend followed by XTE J1814-338 is a spin down taking place over the whole course of the outburst. A spin down during accretion is also showed by another AMSP, XTE J0929-314, which decelerates at a rate $\dot{v}=(-9.2 \pm 0.4) \mathrm{Hz} / \mathrm{s}$ [12]. The most favored effect to explain such a spin down, is the threading of the inner regions of the accretion disc, by a magnetic field that is allowed to expand to the corotation radius $\left(R_{C}=\left(G M / \Omega_{S}^{2}\right)^{1 / 2}\right)$ thanks to a low accretion rate. In this case the torque contribution that comes from the magnetic field lines that close beyond it, and thus spin faster than matter in the disc, may exceed the spin up torque guaranteed by the accretion of matter. Therefore the net resulting effect can be the spin down of the compact object (see e.g. [13],[14]). Rappaport et al. [10] developed a model 
in which the inner disc radius stays fixed at $R_{C}$, no matter if the nominal value of the magnetospheric radius would place the edge of the magnetosphere beyond $R_{C}$. The disc structure is then readjusted in its inner parts by increasing the matter density, in order to match the request of a net angular momentum flux that is pointed outward. According to this model the torque acting on the central object can be expressed as:

$$
\tau=\dot{M}\left(G M R_{C}\right)^{1 / 2}-\gamma \frac{\mu^{2}}{9 R_{C}^{3}},
$$

where the first term is the usual spin up torque owing to the accretion of matter at $R_{C}$, while the second is the magnetic spin down torque, which mainly depends on the value of the magnetic dipole moment $\mu$, and is parametrized by the factor $\gamma$, which is of the order of unity.

An estimate of the bolometric peak flux has been given in [15], leading to an accretion rate $\dot{M}_{\text {peak }}=6 \times 10^{-10} M_{\odot} /$ yr. As during the first 35 days of the outburst the flux remained loosely constant in magnitude and spectral shape, we could consider its average value, and put it into Eq.1, together with the intensity of the torque measured in our timing analysis $(\tau=2 \pi I \dot{v})$, in order to give an estimate of the magnetic dipole moment. In this way we obtain $\mu \simeq 8 \times 10^{26} \gamma^{-1 / 2} \mathrm{G} / \mathrm{cm}^{3}$, which corresponds to a superficial magnetic field $B_{S} \simeq 8 \times 10^{8} \gamma^{-1 / 2} \mathrm{G}$, which is in the correct range $\left(10^{8}-10^{9} \mathrm{G}\right)$ for the AMSP to be the progenitors of the radio millisecond pulsars.

We also note that Romanova et al. [16] performed 3D MHD simulations of the interaction between the accretion disc and the magnetosphere of a NS in the propeller stage (i.e. when the magnetospheric radius is larger than $R_{C}$ ). These result in a continuous spin down of the accretor while accretion proceeds in a cyclic way. The negative torque produced mainly by the predominantly toroidal configuration that the field lines take in the proximity of the disc is strong enough to explain the observed value with similar superficial magnetic field strengths.

\section{REFERENCES}

1. D. Bhattacharya and E. P. J. van den Heuvel, Phys.Rep., 203, 1 (1991)

2. R. Wijnands and M. van der Klis, Nature, 394, 344 (1998)

3. R. Wijnands, Proc. AAS Meeting, 209, 208.3 (2006)

4. C. B. Markwardt, J. H. Swank, IAU Circ., 8144, 1 (2003)

5. K. Jahoda et al., in EUV, X-ray, and Gamma-Ray Instrumentation for Astronomy VII, edited by O. H. Siegmund and M. A. Gummin, Proc. SPIE Vol.2808, SPIE, Washington, 1996, p.59

6. M. I. Krauss et al., ApJ, 627, 910 (2005)

7. L. Burderi et al., ApJ, 657,961 (2007)

8. P. Gosh and F. K. Lamb, in Neutron Stars: Theory and Observation, edited by J. Ventura and D. Pines, NATO ASI Ser., Dordrecht, Kluwer, 1991, p.363

9. L. Burderi et al., ApJ, 498, 831 (1998)

10. S. A. Rappaport, J. M. Fregeau and H. C. Spruit, ApJ, 606, 436 (2004)

11. L. Burderi L. et al., ApJ, 653, L133 (2006)

12. D. K. Galloway et al., ApJ, 576, L137 (2002)

13. P. Gosh and F. K. Lamb, ApJ, 234, 296 (1979)

14. Y.-M. Wang, A\&A, 183, 257 (1987)

15. D. K. Galloway, A. Cumming and D. Chakrabarty, BAAS, 8, 25.01 (2004)

16. M. M. Romanova et al., ApJ, 616, L151 (2004) 
Copyright of AIP Conference Proceedings is the property of American Institute of Physics and its content may not be copied or emailed to multiple sites or posted to a listserv without the copyright holder's express written permission. However, users may print, download, or email articles for individual use. 